\title{
Microblaze Implementation of GPS
}

\author{
Ananya Choudhury ${ }^{1, *}$, Ankith Srinivasan ${ }^{2}$, Aditya R Kattimani ${ }^{3}$, Saroja V \\ Siddamal ${ }^{4}$, Shraddha B Hiremath ${ }^{5}$ \\ 1,2,3,4,5 School of Electronics and Communication Engineering, KLE Technological University, India \\ ${ }^{*}$ Corresponding author. Email: choudhuryananya3099@gmail.com
}

\begin{abstract}
The accuracy and efficiency of a Global Positioning System (GPS) is a critical parameter especially for surveillance and automation purposes. The design of such a system which can not only provide high accuracy and efficiency but also is easily configurable enhances its applications largely for embedded systems. In this paper, the authors have proposed a design of a GPS implementation using a Microblaze which is a Xilinx based Field Programmable Gate Array (FPGA) soft core microprocessor that has over seventy user configurable options, optional Memory Management Unit (MMU) and a five-stage pipeline for maximum performance. The proposed design is used to track and route the path travelled by the user on a map and compute the distance. The Microblaze is used to connect and interface various configurable peripherals like USB UART and LEDs to implement a GPS using the AXI interface of an Arty A7-100T FPGA Evaluation Board. A comparison of the proposed design with respect to the performance parameters has been drawn to analyse the merits and specifications of the system.
\end{abstract}

Keywords: AXI Interface, Field Programmable Gate Array, Global Positioning System, MATLAB, Microblaze.

\section{INTRODUCTION}

The GPS is a widely used application-based device which helps in navigation, location tracking, surveillance and security purposes. This paper provides a GPS implementation on a soft-core processor like Microblaze to achieve high scale performance, efficiency and easily configurable capability. To realize the design, the authors have used the soft-processor of an FPGA (Arty A-7 100T) which makes designing and debugging easier as well as minimizes timing and routing issues.

The system is designed to obtain accurate NMEA data for tracking, routing and computing the distance travelled by the user. The block design is configured using the AXI interface and customizable peripherals on the Xilinx Vivado Design Suite and the GPS signals are transmitted from a mobile sensor to the Memory Management Unit of the system. The design provides the flexibility to configure and modify peripherals and connections for varied applications. The MATLAB Mapping Toolbox is used in routing the path travelled on a Street View Map marking the two end points. The performance parameters like accuracy and sensitivity of the system are analysed and compared with other designs and methods to study and interpret the merits of the proposed design.

\section{RELATED WORKS}

There are several works with different methodologies and designs to implement a GPS system with higher efficiency and varied applications and scopes. The accuracy, speed and versatility of different architectures and designs have also been an important development by the researchers. The design of an FPGA architecture based on parallel pipelining is implemented and compared to other traditional data processing methods. The conclusion of the comparison has shown that the architecture proposed in paper [1] improves the speed of data processing. A study [2] proposes an architecture of an FPGA based location tracking system that interfaces a GPS and a GSM to track the location of a user and transmit the data to a mobile phone. The design of an Artix-7 FPGA based control pulse generation is presented by the authors [3] by communicating between a TR module and a radar controller using the Ethernet PHY. 
The protype model in [4] uses UART soft IP core of a Microblaze on Spartan 3E FPGA to develop serial communication between various systems. The research work displays the status of switches present on the board on HyperTerminal as well as on the LEDs on the board. The serial communication has been carried out using RS232 cable connected between the FPGA board and COM port of PC. For this, the UART soft IP core is designed by the use of the core generator system present in the Xilinx (ISE) design suite. Xilinx's (EDK) is a package designed to build Xilinx FPGAs with Microblaze embedded processor systems. A soft-core Microblaze implemented in a Spartan 3E FPGA is customized by using UART, GPIO, timer and IP core and a GPS receiver is connected with the processor which receives the data and the data is analysed in the processor and decoded, the then output is displayed using UART [5].The process of acquisition of GPS signals using MATLAB and its signal processing tools to receive the GPS Signals has been discussed and implemented on an FPGA by integrating and executing the prototyping and the simulation tools together [6]. A research has been done on synchronizing pulses acquired by GPS units to measure synchronization. The paper [7] also highlights the disadvantages of using GPS and analyses the accuracy of a microcontroller-based system and an FPGA based system. A survey report in paper [8] concludes that satellite navigation is not easily configurable as they are designed by keeping specific use in mind. An easy to configure GPS using FPGA (Altera Cyclone 2) has been designed with multiple satellite connection for real-time tracking to solve this issue.

Issues like accuracy, resource usage of FPGA, latency and power have been given importance and an improvised design is proposed [9] to avoid such problems by using a loosely-coupled version of the circuit and a softcore Microblaze processor. A comparison has been drawn between two ways of implementing a GPS-INS integrated system using a Microblaze. The authors have used two types of FPGAs, one being Spartan and the other being Zynq [10]. The problems incurred in such systems due to accuracy of the position, GPS outages, connectivity to satellites and power consumption have been taken into consideration.

A low-priced two-dimensional RISS (reduced inertial sensor) having a lot of other integrated sensors like gyroscope and odometer is used in the proposed design [11] to transmit GPS signals using a Kalman filter. The design has been used to build and implement a real-time inexpensive yet efficient embedded system for navigation purposes which is also capable of computing the positioning data for various applications by synchronizing the measurements from the three sensors using a soft core Microblaze.

A microcontroller-based GPS/GSM vehicular tracking system has been designed to configure the GPS to track a vehicle and transmit the data to the microcontroller where the GPS data is analysed and decoded to an understandable format and transmitted to another device using a GSM module [12].

To understand the design flexibility of a softcore Microblaze processor, several applications have been discussed and designed. A data of a heart rate senor implemented on a Spartan 3E FPGA board is analysed in the processor with a GSM system that has been integrated with the FPGA using UART serial communication to send real-time messages to a mobile [13]. With the use of different sensors and wireless communication devices interfaced with the configurable peripherals of an FPGA, a system is designed to build an intelligent home control device [14]. The architecture of an FPGA based parking system using Spartan 6 is also an application-based research that offers a versatile design [15].

\section{METHODS}

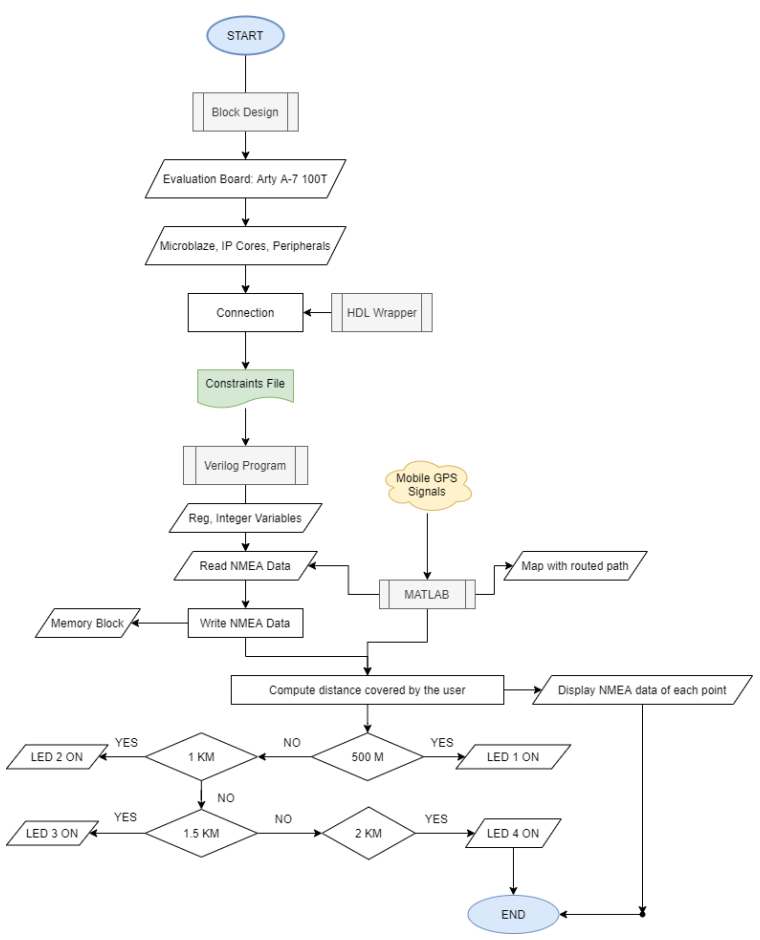

Figure 1 The flow of processes to complete the design.

As shown in Figure 1 a programmable FPGA (Arty A7-100T) is selected for creating the block design. The memory block, Microblaze processor, IP cores and configurable peripherals are added and required connections are made for the interface between different IP Cores. The local and cache 
memory is configured to $16 \mathrm{kB}$ and the block design is validated. The validated design is wrapped by creating the HDL Wrapper for synthesis. The master XDC (Xilinx Design Constraints) file is configured to constrain the input and output pins for the given FPGA board and route the physical pins on the board required for our design [16-20].

Using the mobile GPS sensors, real-time data is sent to the MATLAB drive and using the given data, the MATLAB Mapping Toolbox is programmed to generate a virtual GPS tracking webpage and transmit the NMEA data (Latitude, Longitude) to the memory of the Microblaze through UART. The webpage which is a live view of a map displays the route taken by the user and marks the starting and ending points [21-25].

The FPGA is now programmed using a Verilog code to perform the required tasks. The NMEA data transmitted by the GPS signals of the phone using MATLAB is read and written on the memory of the Microblaze through the UART. Using the location data of the starting and ending points of the route taken by the user, the distance covered is computed using the mathematical functions (sine, inverse cosine) and performing arithmetic operations.

radians $=\frac{\text { degrees } \times \pi}{180}$

Using Equation (1), the values of latitude and longitude of the two end points are converted to radians.

distance $=3963 \times \cos ^{-1}(\sin$ lat $1 * \sin$ lat 2$) \times$ cos lat $1 \times \cos 1$ lat $2 \times \cos (\operatorname{lon} 2-\operatorname{lon} 1)$

Equation (2), gives us the distance between the two end points in miles where lat1, lat 2 are the latitudes (in radians) and lon1, lon2 are the longitudinal values (in radians) respectively.

distance $($ in $\mathrm{m})=1609.34 \times$ distance $($ in miles $)$

Equation (3), gives us the distance between the two end points in meters.

The above computed value is used to check the distance travelled by the user. The behavioural simulation of the design is used to test the proper working of the system, the LEDs act as testers which is set high (glows) after every 500 metres of distance travelled by the user. This will check if the programmed FPGA is working as per the logic. The latitude and longitude at every point of the route is displayed from the memory using a string array which executes till the end point is reached.

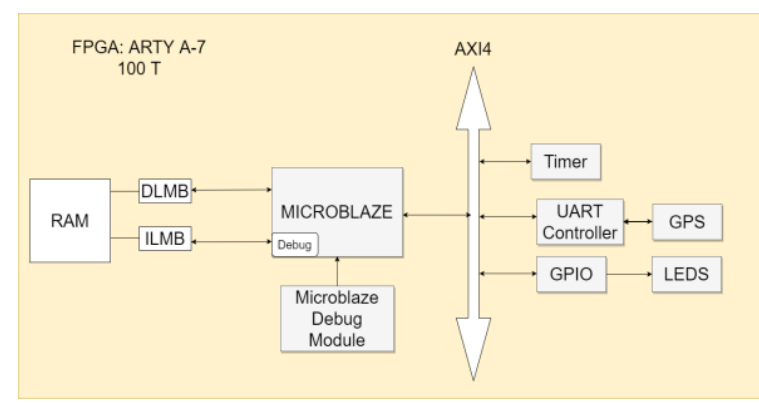

Figure 2 System architecture of the design showing the interfaces between the different components.

Figure 2 shows the communication between the Microblaze and the connected peripherals using AXI4 protocol. The GPS data received using the mobile sensors is transmitted into the memory of the system using the UART controller. The 4 bits LEDs are also interfaced using the general purpose AXI interface which transmits the computed data to the ports.

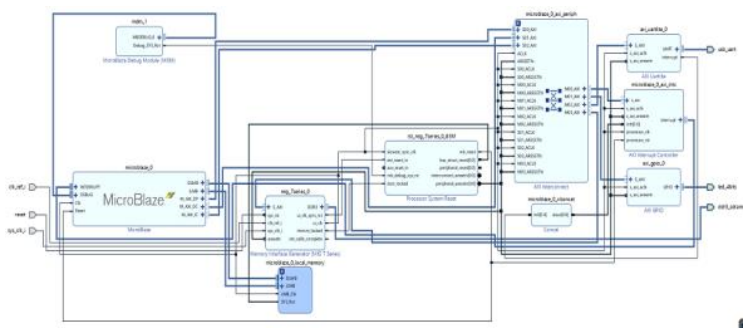

Figure 3 Block diagram of the connections and interfaces of the design.

In Figure 3 an external memory interface, DDR3 SDRAM, is used to generate the memory within the internal logic of the Arty A7-100T FPGA. The 32bits soft core processor, Microblaze with an internal local and cache memory is used to interface with the memory block in Figure 3. The AXI protocol is used to transmit and receive data from the configurable IPs interfaced in the design. One of the required peripherals, USB UART is configured with a slave bus interface to transmit and receive the TX and RX signals to and from the Microblaze Debug Module. The NMEA data acquired from the GPS signals is transmitted through the USB UART and the data is captured and stored in the memory. Another configurable peripheral used for the design are the LEDs which are connected to the general purpose AXI interface to transfer the required data from the port to the GPIOs. The LEDs will be used as route distance testers on the behavioural simulation waveform [26-29]. 


\section{RESULTS}

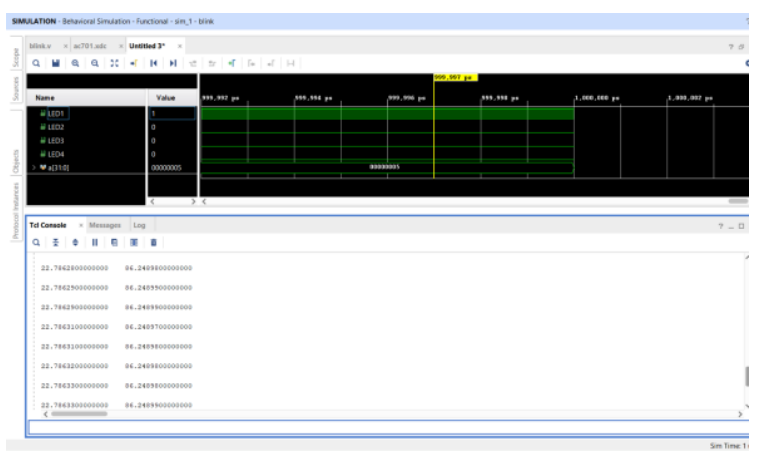

Figure 4 Simulation waveform of the design.

Figure 4 shows the LEDs which are used as markers for every $500 \mathrm{~m}$ travelled. The location (latitude and longitude) of each point throughout the path travelled by the user is displayed on the tcl console. This shows that the MMU of the architecture captures the NMEA data continuously and the same data is used to determine the starting and ending points of the path. The distance covered is computed using the latitude and longitude of the end points as discussed in Section 3. The LEDs are used to validate the computed distance and behaves as markers for every $500 \mathrm{~m}$.

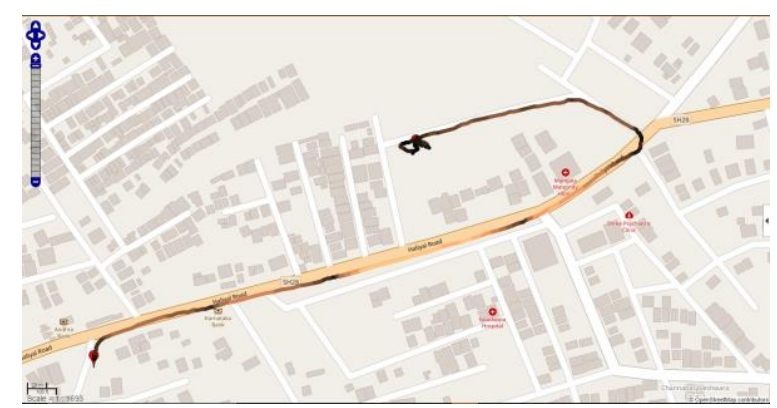

Figure 5 The path travelled by the user is plotted on a map.

Figure 5 displays a street view map using the MATLAB Mapping Toolbox. The GPS signals that are received from the mobile sensors and transmitted to the MMU of the FPGA are loaded simultaneously on MATLAB for data processing and mapping. With the use of a street view map, the path is routed on the map and the starting and ending points are marked. Parameters like colour and width of the map are also set in the program.

\section{DISCUSSION}

\subsection{Optimization}

\subsubsection{USB UART over JTAG}

JTAG interface is a special type of interface on chip, multiple chips on board are connected in star topology, to access these chips JTAG port is used. Whereas USB UART provides USB connectivity to UART devices. The latter has been chosen as it is faster, reaching speeds up to $480 \mathrm{Mbps}$ while JTAG only managing maximum of $1 \mathrm{Mbps}$.

\subsubsection{AXI over PLB}

AXI data bus and address bus are not fixed neither within a given system you can have a wide variety of data bus widths. In AXI one can write data and address in any order. A write address setup is not required to write relevant address. A turn-around is not needed as the write bus is completely separate. In AXI insertion of pipelines is very easy and nonintrusive. One can add as many register slices to the 5 channels with no effect on the final communicated data. With all of these features AXI has a clear advantage over PLB.

\subsection{Comparative Analysis}

Table 1 shows a comparative analysis of different designs and models with respect to the performance measures like horizontal position accuracy, update rate and sensitivity.

Horizontal position accuracy is the radius of a circle drawn around the precise location as at a given point of time a GPS receiver plots multiple positions for a given point, the smaller the radius, higher the accuracy. If we consider our proposed model the GPS being used is that found in modern smartphones which have high accuracy because the radius of the circle may vary from $1.6 \mathrm{~m}$ to $1 \mathrm{~m}$ whereas the rest of the methods have higher radius, lowest being $1.6 \mathrm{~m}$.

Update Rate of a GPS is the number of times the system calculates and computes the location, measured in Hertz. The standard update rate for most devices is $1 \mathrm{~Hz}$. The higher update rate comes with a

Table 1. Comparative analysis of performance measures with different design models.

\begin{tabular}{|l|c|c|c|c|}
\hline \multicolumn{1}{|c|}{ Method } & $\begin{array}{c}\text { Horizontal Position } \\
\text { Accuracy }\end{array}$ & Update Rate (in Hz) & $\begin{array}{c}\text { Number of Channels } \\
\text { Accessible }\end{array}$ & $\begin{array}{c}\text { Sensitivity } \\
\text { (in dBm) }\end{array}$ \\
\hline Proposed Model & $1.6 \mathrm{~m}-1 \mathrm{~m}$ & 1 & $10-16$ & -161 \\
\hline Rao et al. [9] & $1.6 \mathrm{~m}-2.1 \mathrm{~m}$ & $1-10$ & 50 & -161 \\
\hline Farid et al. [11] & $2.5 \mathrm{~m}$ & $1-5$ & 22 & -160 \\
\hline Nkem [12] & $2.5 \mathrm{~m}$ & $1-5$ & 22 & -160 \\
\hline
\end{tabular}


hefty price of more energy consumption as to push out more $\mathrm{Hz}$ more energy is required similar to the refresh rate of $\mathrm{TV} /$ monitor, $1 \mathrm{~Hz}$ is more than capable to track a moving object up to speeds reaching 75 Kmph so the use of a higher update rate GPS won't be necessary for the project for the time being.

All the satellites transmit the same frequency, so the GPS mixes up the signals causing confusion. To fix this problem, channels are used. A single channel correlates the signals (more the channels faster the process) and segregates the information. Higher channel GPSs are really expensive compared to lesser channel ones. Point to be noted that a GPS having higher Update rate will require more channels to process the information.

A GPS having a higher sensitivity is able to track very weak GPS signals which in turn increases the accuracy of the location information, considering the proposed model up to $-161 \mathrm{dBm}$. The other methods have similar sensitivity.

\section{CONCLUSION}

In this paper, Microblaze of an FPGA, Arty A7$100 \mathrm{~T}$ has been designed, programmed and simulated for a GPS implementation using configurable peripherals and memory interfaces. The system is realized by integrating the Microblaze, IP cores and peripherals of the FPGA to communicate with the mobile sensor GPS for accurate location and positioning using the MATLAB tools. The path taken by the user is routed on a map and the distance covered is computed. The calculated distance is tested by switching the LEDs of distinct colours, interfaced with the Microblaze, on and off for every 500 metres covered by the user. The location (NMEA data) at each point throughout the path is captured in the memory and displayed at the simulation. The design can be used to communicate and integrate with several GPS sensors and these data can be controlled and implemented for various applications. It will provide a scope of computing relative data among several automobiles, mobile phone users for measuring and comparing the relative distance, routes and paths, intersecting points. Additional sensors can also be implemented on the Microblaze using this design as a base. The GPS implemented Microblaze can be used in various real-time embedded systems for navigation and surveillance purposes. The FPGA can be implemented with other processors on automobiles as it will not only increase the efficiency and performance of the system but also give the ability to customize. The design can be also used for surveillance and security purposes to track and navigate targets or victims.

\section{AUTHORS' CONTRIBUTIONS}

Author 1: Conceptualization, Methodology, Software. Author 2: Methodology, Software, Formal analysis. Author 3: Resources, Writing- Original Draft preparation. Author 4: Validation, Supervision. Author 5: Supervision

\section{ACKNOWLEDGMENTS}

We would like to thank our university for giving us a chance to work on such a unique project and for providing us the necessary resources and guiding us at every step which paved our way towards the successful completion of the paper.

\section{REFERENCES}

[1] Wei He, Zhi Jiang, Zengshan Tian, Mu Zhou, Shuai Lu, FPGA Design and Optimization Implementation of GPS Positioning Algorithm, School of Communication and Information Engineering, Chongqing University of Posts and Telecommunications, Mobimedia 2020, 27-28 August 2020, Cyberspace. DOI: https://doi.org/10.4108/eai.27-8-2020.2295315

[2] Govindaraju Kavya and Vittal Thulasi Bai, Design and Implementation of Global Positioning System Receiver in Field Programmable Gate Array with Short Message Service, Journal of Computer Science 10 (1): 91-98, 2014 ISSN: 1549-3636. DOI: 10.3844/jcssp.2014.91.98

[3] P Kamaraj and R Jagadeesh Kannan, Real Time Design and Implementation of FPGA Based Timing and Control System for Wireless Radar Communication, IOP Conf. Series: Materials Science and Engineering 925 (2020) 012064. DOI: $10.1088 / 1757-899 X / 925 / 1 / 012064$

[4] Sanmati Patil, Rajanish Kamat, Pawan Gaikwad, FPGA-Based Implementation of MicroBlaze Processor as UART Soft IP Core, International Journal of Innovative Research in Science, Engineering and Technology (IJIRSET), volume 9, issue 7, July 2020, eISSN: 2319-8753, p-ISSN: 2320-6710.

[5] Wang Ershen, Zhang Shufang, Zhang Zhixian, Research on Embedded GPS Reciever Based on Xilinx Microblaze, in: Communications Technology, Vol.43, No.10,2010.

[6] Mohamed EL Hawary, Abdelhalim abdelnaby Zekry, FPGA Implementation of Acquisition Phase of the GPS Receiver Using XSG, National Institute of Standards (Egypt), International Journal of Electronics and Telecommunications, October 2019, Vol. 65, 
No. 4, Pp. 739-744. DOI: 10.24425/ijet.2019.130257

[7] Zdenek Slanina, Vladimir Kasik, Karel Musil, GPS Synchronisation for FPGA Devices, IFAC Proceedings Volumes, Volume 45, Issue 7, 2012. DOI: https://doi.org/10.3182/20120523-3CZ-3015.00064

[8] Adam M. Shapiro, FPGA-Based Real-Time GPS Receiver, Engineering Division of the Graduate School of Cornell University, January, 2010, Semantics Scholar, Corpus ID: 17271424.

[9] Lokeswara Rao Bhogadi, Sasi Bhushana Rao Gottapu and VVS Reddy Konala, MicroBlaze implementation of GPS/INS integrated system on Virtex-6 FPGA, Bhogadi et al. SpringerPlus (2015) 4:629. DOI: 10.1186/s40064-015-1367-y

[10] B.Lokeswara Rao, Associate Professor, Dr.K.V.V.S Reddy, Former Professor, Dr.G.Sasi Bhushana Rao, Professor HOD, Performance Analysis of Microblaze Implemented GPS-INS Integrated Systems on Spartan 6 and Zynq FPGAs, IOSR Journal of Electronics and Communication Engineering (IOSR-JECE) e-ISSN: 2278-2834,p- ISSN: 2278-8735.Volume 9, Issue 2, Ver. VI (Mar Apr. 2014), PP 01-13.

[11] Walid Farid Abdelfatah, Jacques Georgy, Umar Iqbal and Aboelmagd Noureldin, FPGA-Based Real-Time Embedded System for RISS/GPS Integrated Navigation, Sensors 2012, 12, 115147. DOI: $10.3390 / \mathrm{s} 120100115$

[12] Nwukor Frances Nkem, Implementation of Car Tracking System using GSM/GPS, Electrical and Electronic Engineering Department, Petroleum Training Institute, Effurun, Delta State, Nigeria, International Journal of Scientific and Research Publications, Volume 10, Issue 3, March 2020.

[13] P. K. Gaikwad, Development of FPGA Microblaze Processor and GSM Based Heart Rate Monitoring System, International Journal of Computer Science and Mobile Applications, Vol.1 Issue. 3 September- 2013.

[14] Tian Hua, Design and Implementation of Smart Home Control System Based on FPGA, 2020 Frontiers in Educational Research, ISSN 25226398 Vol. 3, Issue 13: 68-72. DOI: 10.25236/FER.2020.031311

[15] S.Sharmila Devi , Blessy Angel J.J.R., Deepa.M., Kaaviya.A.I., Car Parking System Using FPGA, International Research Journal on Advanced Science Hub (IRJASH), International Research Journal on Advanced Science Hub (IRJASH), e-ISSN: 2582-4376.
[16] Bhuvaneswary, N., S. Prabu, S. Karthikeyan, R. Kathirvel, and T. Saraswathi. "Low Power Reversible Parallel and Serial Binary Adder/Subtractor." Further Advances in Internet of Things in Biomedical and Cyber Physical Systems (2021): 151.

[17] Bhuvaneswary, N., S. Prabu, K. Tamilselvan, and K. G. Parthiban. "Efficient Implementation of Multiply Accumulate Operation Unit Using an Interlaced Partition Multiplier." Journal of Computational and Theoretical Nanoscience 18, no. 4 (2021): 1321-1326.

[18] Do, Dinh-Thuan, Tu Anh Le, Tu N. Nguyen, Xingwang $\mathrm{Li}$, and Khaled M. Rabie. "Joint impacts of imperfect CSI and imperfect SIC in cognitive radio-assisted NOMA-V2X communications." IEEE Access 8 (2020): 128629-128645.

[19] Le, Ngoc Tuyen, Jing-Wein Wang, Duc Huy Le, Chih-Chiang Wang, and $\mathrm{Tu}$ N. Nguyen. "Fingerprint enhancement based on tensor of wavelet subbands for classification." IEEE Access 8 (2020): 6602-6615.

[20] Naeem, Muhammad Ali, Tu N. Nguyen, Rashid Ali, Korhan Cengiz, Yahui Meng, and Tahir Khurshaid. "Hybrid Cache Management in IoTbased Named Data Networking." IEEE Internet of Things Journal (2021).

[21] Subramani, Prabu, K. Srinivas, R. Sujatha, and B. D. Parameshachari. "Prediction of muscular paralysis disease based on hybrid feature extraction with machine learning technique for COVID-19 and post-COVID-19 patients." Personal and Ubiquitous Computing (2021): 1-14.

[22] Kumar, M. Keerthi, B. D. Parameshachari, S. Prabu, and Silvia liberata Ullo. "Comparative Analysis to Identify Efficient Technique for Interfacing BCI System." In IOP Conference Series: Materials Science and Engineering, vol. 925, no. 1, p. 012062. IOP Publishing, 2020.

[23] Rajendrakumar, Shiny, and V. K. Parvati. "Automation of irrigation system through embedded computing technology." In Proceedings of the 3rd International Conference on Cryptography, Security and Privacy, pp. 289-293. 2019.

[24] Z. Guo, L. Tang, T. Guo, K. Yu, M. Alazab, A. Shalaginov, "Deep Graph Neural Networkbased Spammer Detection Under the Perspective of Heterogeneous Cyberspace", Future Generation Computer Systems, https://doi.org/10.1016/j.future.2020.11.028. 
[25] Y. Sun, J. Liu, K. Yu, M. Alazab, K. Lin, "PMRSS: Privacy-preserving Medical Record Searching Scheme for Intelligent Diagnosis in IoT Healthcare", IEEE Transactions on Industrial Informatics, doi: 10.1109/TII.2021.3070544.

[26] N. Shi, L. Tan, W. Li, X. Qi, K. Yu, "A Blockchain-Empowered AAA Scheme in the Large-Scale HetNet", Digital Communications and Networks, https://doi.org/10.1016/j.dcan.2020.1 0.002 .

[27] C. Feng et al., "Efficient and Secure Data Sharing for 5G Flying Drones: A BlockchainEnabled Approach," IEEE Network, vol. 35, no. 1, pp. 130-137, January/February 2021, doi: 10.1109/MNET.011.2000223.

[28] L. Tan, H. Xiao, K. Yu, M. Aloqaily, Y. Jararweh, "A Blockchain-empowered Crowdsourcing System for 5G-enabled Smart Cities", Computer Standards \& Interfaces, https://doi.org/10.1016/j.csi.2021.10 3517

[29] Z. Guo, Y. Shen, A. K. Bashir, M. Imran, N. Kumar, D. Zhang and K. Yu, "Robust Spammer Detection Using Collaborative Neural Network in Internet of Thing Applications", IEEE Internet of Things Journal, vol. 8, no. 12, pp. 9549-9558, 15 June15, 2021, doi: 10.1109/JIOT.2020.3003802. 